Cahiers $d u$ MONDE RUSSE

\section{Cahiers du monde russe}

Russie - Empire russe - Union soviétique et États indépendants

$44 / 4 \mid 2003$

Varia

\title{
V. N. Zemskov, Specposelency v SSSR, 1930-1960
}

\section{Catherine Gousseff}

\section{OpenEdition}

\section{Journals}

Édition électronique

URL : https://journals.openedition.org/monderusse/4115

DOI : 10.4000/monderusse.4115

ISSN : $1777-5388$

\section{Éditeur}

Éditions de l'EHESS

\section{Édition imprimée}

Date de publication : 1 octobre 2003

Pagination : 740-742

ISBN : 2-7132-1833-0

ISSN : $1252-6576$

\section{Référence électronique}

Catherine Gousseff, «V. N. Zemskov, Specposelency v SSSR, 1930-1960 », Cahiers du monde russe [En ligne], 44/4 | 2003, mis en ligne le 19 juin 2009, consulté le 04 septembre 2022. URL : http:// journals.openedition.org/monderusse/4115; DOI : https://doi.org/10.4000/monderusse.4115

Ce document a été généré automatiquement le 4 septembre 2022

Tous droits réservés 


\title{
V. N. Zemskov, Specposelency v SSSR, 1930-1960
}

\author{
Catherine Gousseff
}

\section{RÉFÉRENCE}

\section{N. ZEMSKOV, Specposelency v SSSR, 1930-1960. Moscou, Nauka, 2003, 306 p.}

Dans la nouvelle historiographie "post-soviétique » qui a bénéficié de l'ouverture des archives, les recherches de V.N. Zemskov sur les " déplacés spéciaux " (specposelency) se sont imposées dès le début des années 1990 comme des travaux de référence sur l'organisation du système répressif stalinien et la diversité des pratiques punitives. En dehors de l'univers concentrationnaire du travail forcé, les déportations de masse et la création de peuplements spéciaux auxquelles elles donnèrent lieu sur l'ensemble du territoire soviétique ont été l'une des pratiques répressives les plus développées depuis le carrefour des années 1920 et 1930 à l'encontre d'individus et de groupes jugés indésirables, "socialement étrangers ", suspects, nuisibles, etc. À travers cet ouvrage, V. N. Zemskov propose une synthèse de l'histoire des déportés ou "déplacés spéciaux ", effectuée presqu'exclusivement d'après les sources administratives soviétiques, et en particulier les archives du Département des peuplements spéciaux du NKVD-MVD-Gulag.

2 Les «déplacés spéciaux» constituent une catégorie administrative qui recouvre en réalité d'autres désignations usitées au cours de la période, telles que "colons du travail » (trudposelency), ou « déportés spéciaux» (specpereselency). Du point de vue de leur statut, les « déplacés spéciaux » ont, théoriquement du moins, conservé leurs droits civiques tout en étant assignés à résidence. Au-delà de cette situation commune, la durée de l'exil, les conditions d'existence, de travail, d'accès à l'éducation, ont beaucoup varié d'un groupe de réprimés à l'autre tout au long de la période stalinienne.

3 Cette rétrospective historique est caractérisée par un intense souci d'exhaustivité. En retraçant l'histoire des "déplacés spéciaux", V.N. Zemskov aborde, au fil de la chronologie, toutes les opérations de déportations, depuis celles, majeures, qui ont 
affecté les « koulaks ", jusqu'aux groupes de «nationalistes », de rapatriés de l'aprèsguerre, sans pour autant négliger les actions, numériquement parlant de faible envergure, telle la déportation, au printemps 1951, de 70 Polonais en provenance de Pologne. Sans chercher à démêler les faits déjà connus, de ceux que révèlent les archives, l'auteur s'attache surtout à rendre compte de toutes les informations livrées par les sources, abreuvant le lecteur de très nombreux chiffres, statistiques, tableaux qui jalonnent un texte lui-même très peu distancié des documents.

4 Dans le chapitre consacré à la première grande vague de déportations liée à la dékoulakisation, et qui toucha 1800000 personnes en 1930-1931, l'historien montre la très importante mortalité enregistrée dans ces contingents, l'origine géographique des déportés, dont les trois quarts venaient de RSFSR ( $16 \%$ d'Ukraine), et leur répartition sur le territoire - l'Oural et la Sibérie occidentale concentrant plus de la moitié d'entre eux en 1933 -, leur niveau d'éducation (les trois quarts n'ayant reçu qu'une éducation élémentaire), etc. Il retrace la normalisation progressive de la situation des déportés dans la deuxième moitié de la décennie, à travers la diminution de la mortalité, la relative uniformisation des modalités de gestion, les mesures de libération qui touchèrent une partie des koulaks et notamment celle de 1938 qui a concerné les enfants de déportés. Dans cette période, Zemskov constate l'extraordinaire complexité des situations liées, en particulier, à la présence de plus en plus importante de citoyens libres dans les colonies, aux mariages « mixtes » entre libres et déportés. En 1938, il existe 1741 colonies de travail comptant $48 \%$ d'actifs. À cette date, les contingents de réprimés se sont considérablement diversifiés, reflétant la politique d'épuration des grandes villes qui a accompagné la passeportisation et surtout la montée des répressions ethniques engagées à l'égard des minorités frontalières qui préfigure la déportation des peuples durant la guerre. Néanmoins, jusqu'à la fin de 1940, les koulaks représentent encore $90 \%$ de la population constituée par les déplacés spéciaux.

5 La déportation de près d'un million d'Allemands à partir de 1941 marque un tournant ; elle sera suivie, au cours de la guerre, de nombreuses autres opérations visant les Grecs, les Finnois, sans compter, à partir de 1944, les peuples du Nord Caucase (Tchétchènes, Ingouches), les Tatars de Crimée et, dès le printemps de la même année, les familles des membres de l'Organisation nationaliste ukrainienne (OUN). Si les koulaks furent réprimés sans que soit précisée la durée de leur exil, les déportés au cours de la guerre le furent «à perpétuité ». Parallèlement à la rétrospective de ces nouvelles vagues de répressions, Zemskov évoque la mobilisation des "déplacés spéciaux " dans l'Armée rouge qui concernèrent environ 35000 hommes, dont une partie fut, par la suite, médaillée.

6 À la fin de la guerre, 2200000 personnes sont enregistrées comme " déplacés spéciaux ", dont plus de 800000 sont localisées au Kazakhstan. La mortalité est neuf fois plus élevée en moyenne que la natalité mais cette proportion varie considérablement selon les nationalités, les différents groupes du Nord Caucase enregistrant les taux de mortalité les plus importants. Dans l'après-guerre, on assiste à un chassé-croisé entre la libération, quasi intégrale, des koulaks de 1946 à 1949, et l'arrivée de nouveaux contingents, largement dominés par les groupes en provenance des nouveaux territoires annexés de l'Ouest, mais qui comprend également les Vlassoviens (près de 113000 personnes), des rapatriés de l'étranger, dont des émigrés de longue date, des Iraniens apatrides de Géorgie en 1950, etc. 
7 Dans la rétrospective de cette période, l'auteur s'attache à restituer, à l'appui de différents rapports, les comportements politiques des déportés. Ainsi, dans le plaidoyer développé par certaines autorités des républiques en faveur de la libération de contingents d'anciens koulaks, la loyauté des déportés pendant la guerre, leur fidélité à la patrie est l'un des arguments les plus fréquemment invoqués. Plus généralement, de nombreux groupes de déportés - à l'exception notable des Vlassoviens ou des nationalistes ukrainiens - ont manifesté leur attachement à la "patrie soviétique " notamment à travers leur entrée dans les Komsomols.

8 Il est regrettable de ne pouvoir développer davantage les nombreux éclairages fournis par cet ouvrage, dans des domaines aussi divers que la politique de passeportisation, évoquée selon les contingents et à différentes périodes, le long et incomplet processus de libération de 1954 à 1960, l'alternance des mesures libérales et contraignantes à l'égard des mineurs, etc. Il faut néanmoins signaler aussi les lacunes de l'étude. Ainsi le personnel d'encadrement et de contrôle, et, de manière plus générale, l'organisation de la gestion administrative des colonies sont à peine esquissés, sinon par quelques données sur le personnel égrainées au long de l'ouvrage. La difficulté éprouvée à estimer les écarts entre droits théoriques et contraintes effectives ayant pesé sur les déportés suggère les nondits des sources exploitées. En définitive, le parti pris de Zemskov d'une narration très peu critique des archives montre à la fois la richesse et les limites d'une telle démarche. Par l'importance et la qualité des informations qu'il livre, Specposelency constitue un incontestable ouvrage de référence. Mais il souligne par défaut l'impérieuse nécessité de diversifier les sources pour "réincarner» la part sombre et tragique $d u x^{e}$ siècle soviétique. 